\title{
Multi-Point Contact Models for Dynamic Self-Righting of a Hexapod ${ }^{\star}$
}

\author{
Uluc Saranli ${ }^{1}$, Alfred A. Rizzi ${ }^{1}$, and Daniel E. Koditschek ${ }^{3}$ \\ 1 Robotics Institute, Carnegie Mellon University, Pittsburgh, PA 15213, USA \\ 2 Dept. of EECS, The University of Michigan, Ann Arbor, MI 48109-2110, USA
}

Summary. In this paper, we report on the design of a model-based controller that can achieve dynamical self-righting of a hexapod robot. Extending on our earlier work in this domain, we introduce a tractable multi-point contact model with Coulomb friction. We contrast the singularities inherent to the new model with other available methods and show that for our specific application, it yields dynamics which are well-defined. We then present a feedback controller that achieves "maximal" performance under morphological and actuation constraints, while ensuring the validity of the model by staying away from singularities. Finally, through systematic experiments, we demonstrate that our controller is capable of robust flipping behavior.

Key words: legged robot, model based control, contact modeling, flipping, RHex

\section{Introduction}

RHex is an autonomous hexapod robot that negotiates badly irregular terrain at speeds better than one body length per second [12]. In this paper, we report on efforts to extend RHex's present capabilities with a self-righting controller. Based on our earlier work in this domain [13, 14], we introduce a multi-point contact model based on Coulomb friction so as to derive the maximum benefit of our robot's limited power budget. In designing a model-based feedback controller, we highlight fundamental singularity problems in handling multiple intermittent contacts under external actuation and illustrate a particular solution for our application domain. In particular, we present experimental results that demonstrate the practical implementation of a controller based on the model which yields a reliable self-righting maneuver.

The primary motivation for this problem is to achieve autonomous operation of robotic platforms in unstructured environments. In addition to physical

${ }^{\star}$ Portions of the material in this paper, in combination with material excerpted from [14] have been submitted for publication to the International Journal of Robotics Research. 
necessities such as onboard power and computation, the survivability of such robots in the real world requires at least basic manueuverability capabilities to remain functional in the absence (or inattention) of a human operator [1]. The problem of recovering correct body orientation is one of the simplest among such tasks and constitutes the focus of the present paper.

RHex's morphology is roughly symmetric with respect to the horizontal plane, and allows nearly identical upside-down or right-side up operation, a solution adopted by other mobile platforms [7]. However, many application scenarios such as teleoperation and vision based navigation entail a nominal orientation arising from the accompanying instrumentation and algorithms. In order to secure self-righting capabilities in such settings, most robotic platforms manipulate their body orientation through special kinematic structures (for example, long extension arms or reconfigurable wheels $[3,4,18]$ ). In contrast, the imperatives of dynamical operation that underly RHex's design and confer its unusual mobility performance [12] preclude such structural appendages. RHex must rely on its existing morphology and dynamic maneuvers to achieve the necessary self-righting ability.

In this light, the central contributions of this paper include: i) introducing a frictional multiple point collision/contact model that characterizes RHex's behavior during the flipping maneuver; and ii) the description of a new torque control strategy that uses the model to maximize the energy injected into the system in the face of these constraints (i.e., consistent with maintaining a set of postural invariants integral to the task at hand). We contrast analytical properties of the newly introduced model to a number of other approaches proposed in our earlier work and highlight fundamental problems in their application to the design of feedback controllers. We also present experimental evidence to establish the validity of the present model.

\section{Flipping with RHex}

RHex is a relatively simple hexapod robot with only six actuators, directly coupled to individual passively compliant legs. The rotation axes for the legs are all parallel and aligned with RHex's transverse horizontal body axis (see Fig. 1). Consequently, the most natural backflip strategy for RHex pivots the body around one of its endpoints. Pitching the body in this manner, while keeping one of the body endpoints in contact with the ground, maximizes contact of the legs with the ground for the largest range of pitch angles and thus promises to yield the best utilization of available actuation. In contrast, flipping by producing a sideways rolling motion suffers from early liftoff of three legs on one side as well as the longer protrusion of the middle motor shafts.

For surfaces with sufficiently low lateral inclination, RHex's lateral symmetry and the line of body contact restrict this flipping motion to the saggital plane, admitting the use of planar models for its analysis. 


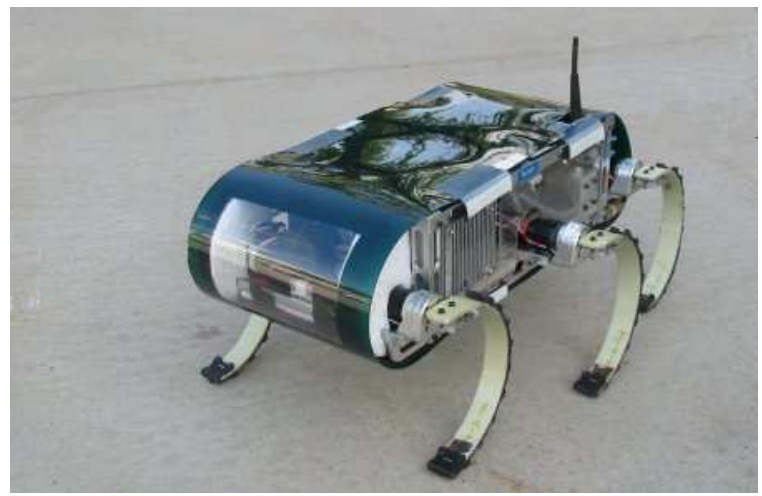

Fig. 1. RHex 1.5

\subsection{Basic Controller Structure}

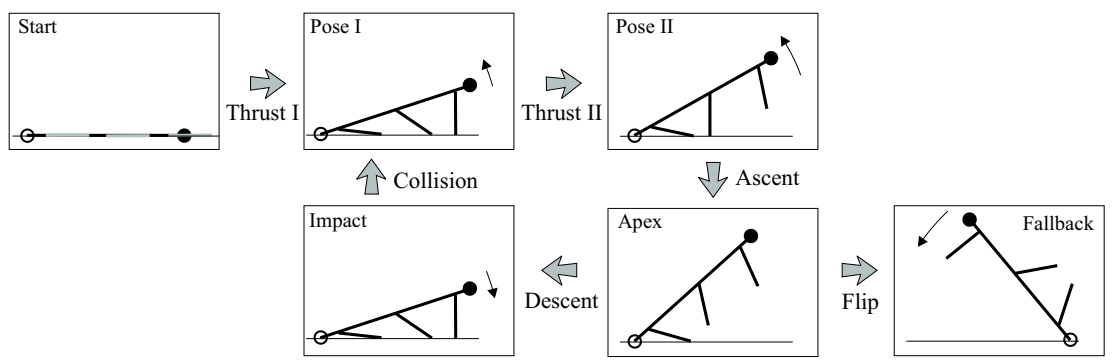

Fig. 2. Sequence of states for the flipping controller

The model-based controller we present in this paper has a finite state machine structure, illustrated in Fig. 2. Starting from a stationary position on the ground, the robot quickly thrusts itself upward while maintaining contact between the ground and the endpoint of its body (poses I and II in Figure 2) as the front and middle legs successively leave the ground. This thrust results in some initial kinetic energy of the body that, depending on the frictional properties of the leg/ground contact, may be sufficient to allow "escape" from the gravitational potential well of the initial configuration, allowing the robot to fall into the desired configuration. In cases where a single thrust is not sufficient to flip the body over, the robot reaches some maximum pitch, and falls back toward its initial state. Our controller then brings the legs back to Pose I of Figure 2 and waits for the impact of the front legs with the ground, avoiding negative work - a waste of battery energy given the familiar power-torque properties of RHex's conventional DC motors. The 
impact of the compliant front legs with the ground in their kinematically singular configuration recovers some of the body's kinetic energy, followed by additional thrust from the middle and back legs, during the period of decompression and flight of the front leg - i.e., during a phase interval when it is possible for the legs in contact to perform positive work on the robot's mass center. The maximum pitch attained by the body increases with each bounce up until the point where the robot flips or the energy that can be be imparted by the thrust phase balances collision losses at which point it must follow that flipping is not possible.

\section{Planar Flipping Models}

\subsection{Assumptions and Constraints}

Two assumptions constitute the basis for our modeling and analysis of the flipping behavior.

First, we assume that the flipping behavior is primarily planar. The controller structure described in Sec. 2.1 operates contralateral pairs of legs in synchrony. On flat terrain, the robot's response lies almost entirely in the saggital plane and departures are rare enough to be negligible. Our models and analysis are thus constrained to the saggital plane.

Second, we assume that the tail of the body should maintain contact with the ground throughout the flipping action. This is motivated by a number of observations gathered during our empirical flipping experiments. During the initial thrust phases, the front and middle legs provide most of the torque. Configurations where the tail endpoint of the body is in contact with the ground yield the longest duration of contact for these legs, harvesting greatest possible benefit from the associated actuators. Collisions of the body with the ground, which introduce significant losses due to the high damping in the body structure designed to absorb environmental shocks, can also be avoided by preserving contact with the ground throughout the flipping action. Finally, the body ground contact is essential for maintaining the planar nature of the behavior and eliminating body roll. This is especially important for repeated thrust attempts of the hybrid energy pumping scheme, which rely on the robot body being properly aligned with as much of the impact kinetic energy recovered as possible.

In light of these assumptions, the design of thrust controllers has to satisfy two major constraints: keeping the tail endpoint of the body on the ground and respecting the torque limitations of the actuators.

\subsection{Generic Planar Flipping Model}

In this section, we introduce a generic planar model that constitutes the basis for our presentation for a number of more constrained variations. 


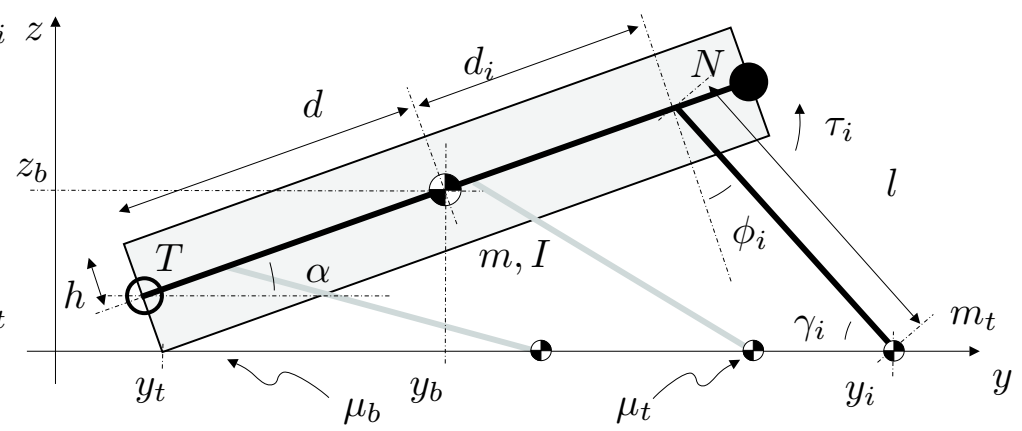

Fig. 3. Generic 3DOF planar flipping model

Figure 3 illustrates the model. Three rigid legs with small toe masses $m_{t}$ - each representing a pair of RHex's legs - are attached to a rectangular rigid body with mass $m$ and inertia $I$. The attachment points of the legs are fixed at $d_{i}$, along the midline of the rectangular body. This line also defines the orientation of the body, $\alpha$, with respect to the horizontal. The center of mass is midway between the points $N$ and $T$, defined to be the "nose" and the "tail". The body length and height are $2 d$ and $2 h$. Finally, the body-ground and toe-ground contacts experience Coulomb friction with coefficients $\mu_{b}$ and $\mu_{t}$, respectively. Table 1 summarizes the notation used throughout the paper.

\begin{tabular}{|l|l|}
\hline \multicolumn{2}{|c|}{ States and dependent variables } \\
\hline$y_{b}, z_{b}, \alpha$ & Body center of mass coordinates and pitch \\
\hline $\mathbf{c} \in \mathcal{X}$ & System configuration vector \\
\hline $\mathbf{q}:=[\mathbf{c}, \dot{\mathbf{c}}]^{T}$ & System state vector \\
\hline$\phi_{i}, \gamma_{i}$ & Hip and toe angles for $i^{t h}$ leg \\
\hline$y_{i}, \dot{y}_{i}$ & position and velocity of the $i^{t h}$ toe \\
\hline \hline \multicolumn{2}{|c|}{ Forces and model parameters } \\
\hline $\boldsymbol{\tau} \in \mathbb{R}^{3}$ & Hip torque control vector \\
\hline $\mathcal{T}(\mathbf{q}) \subseteq \mathbb{R}^{3}$ & Set of allowable torque vectors \\
\hline$F_{i}^{y}, F_{i}, F_{c}^{y}, F_{c}^{z}$ & GRF components on $i^{t h}$ toe and the tail \\
\hline \hline$d, h, m_{,} I$ & Body length, height, mass and inertia \\
\hline$d_{i}, l, m_{t}$ & Leg attachment offset, leg length and leg mass \\
\hline$\mu_{t}, \mu_{b}$ & Coulomb coeff. for toes and body \\
\hline
\end{tabular}

Table 1. Notation used throughout the paper

Our model requires both endpoints of the body as well as the toes to be above the ground, and that a leg must reach the ground before it can apply any torque to the body. We assume that the inertial effects of the toe masses on the body during their flight phase is negligible. 


\subsection{Framework and Definitions}
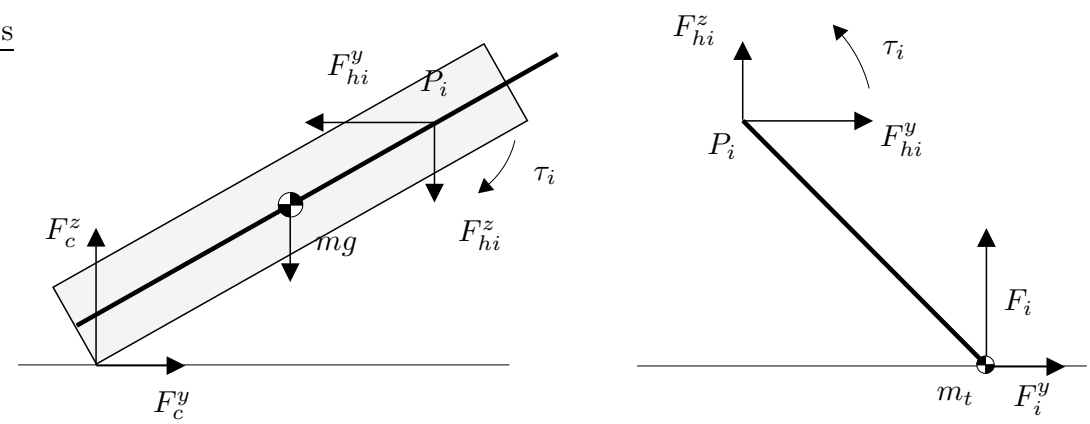

Fig. 4. Free body diagrams for the body and one of the legs

We use a Newton-Euler formulation in deriving the equations of motion for all constrained models. Figure 4 illustrates the free-body diagrams for the body link and one of the leg links. Based on whether a link is in flight, in fixed contact with the ground or sliding on the ground, the associated force and moment balances yield linear equations in the unknown forces and accelerations, taking the form

$$
\mathbf{A}(\mathbf{c}) \mathbf{v}=\mathbf{b}(\mathbf{c}, \dot{\mathbf{c}})+\mathbf{D}(\mathbf{c}) \boldsymbol{\tau} .
$$

The definitions of the configuration vector $\mathbf{c}$ and the vector of unknown forces $\mathbf{v}$, as well as the matrices $\mathbf{A}(\mathbf{c}), \mathbf{b}(\mathbf{c}, \dot{\mathbf{c}})$ and $\mathbf{D}(\mathbf{c})$ are dependent on the particular contact state and will be made explicit in subsequent sections.

\subsection{Simple Model with No Friction and No Toe Mass}

As described in Sec. 2.1, flipping structurally relies on the slipping of the toes and the body to achieve thrust. The simplest model that comes to mind is a rigid body with massless and frictionless legs (i.e. $m_{t}=0, \mu_{t}=0$ and $\left.\mu_{b}=0\right)$. This yields a one degree of freedom system where the ground reaction forces and thus the motion of the center of mass is strictly vertical. The configuration and unknown force vectors are defined as $\mathbf{c}:=\alpha$ and $\mathbf{v}:=\left[F_{1}, F_{2}, F_{3}, \ddot{\alpha}, F_{c}^{z}\right]^{T}$, respectively.

Unfortunately, in trying to derive the dynamics of such a system through a Newton-Euler analysis, one quickly realizes that configurations in which any one of the legs is vertical are singular and yield infinite ground reaction forces. Intuitively, this singularity arises from the faulty assumption that we can apply nonzero torque against a massless leg where the only external force is the vertical ground reaction force. Clearly, this model is physically inaccurate and is hence inappropriate for the analysis of the flipping behavior. 


\subsection{Extended Model with No Toe Friction and Small Toe Masses}

Based on the observations of the previous section, we attempt to add small masses at the toes to counteract the action of the hip torque Assuming sufficient friction the body-ground contact and zero friction at the toes, this results in a one degree of freedom system. Defining the configuration and force vectors as $\mathbf{c}:=\alpha$ and $\mathbf{v}:=\left[F_{1}, F_{2}, F_{3}, \ddot{\alpha}, F_{c}^{z}, F_{c}^{y}\right]^{T}$, the moment balance for leg $i$ takes the form

$$
\left(l \cos \gamma_{i}+l \bar{\mu}_{i} \sin \gamma_{i}\right) F_{i}+\left(l m_{t} b_{i}^{y} \sin \gamma_{i}\right) \ddot{\alpha}=l m_{t} a_{i}^{y} \sin \gamma_{i}-\tau_{i},
$$

where $\bar{\mu}_{i}:=-\mu_{t} \operatorname{sign}\left(\dot{y}_{i}\right)$ and $\ddot{y}_{i}=a_{i}^{y}-b_{i}^{y} \ddot{\alpha}$ is obtained by differentiating the kinematic equations for the toe position $y_{i}$ twice. Note that the contact states of the toes no longer depend only on the configuration, but also on the velocities in the system due to the coriolis terms in $a_{i}^{y}$ as well as the coupling with the pitch acceleration through $b_{i}^{y}$. This primarily results from the fact that the hip torque necessary to produce the horizontal toe acceleration required to maintain contact goes to infinity as the leg approaches its vertical configuration. As a consequence, the leg must "lift off" before it reaches its vertical configuration, at an angle which is a function of both the pitch angle and the pitch velocity. Detailed discussions of the algorithms necessary to uniquely identify contact states for this model and the derivation of its dynamics can be found in [13].

For this model, the ability to uniquely determine the contact state allows us to use the framework of Sec. 3.3 for computing the unknown forces. Surprisingly, there are still configurations for which the matrix $\mathbf{A}(\mathbf{c})$ may become singular. These singularities are much less intuitive and we have not been able to identify a physical explanation for their characterization. Even though for restricted ranges of operation on a particular morphology (such as flipping with RHex) it is possible to show that the system stays away from these singularities, they seem to be "inherent" to the family of models we have investigated and persist even when we reintroduce toe friction. A unifying explanation for this family of singularities remains an open question.

In any case, both the nonlinear kinematics of toe positions and the algorithmic complexities in determining contact state significantly impair the practicality of this model for an embedded implementation. Even though we previously showed simulation evidence for the performance of the model [14], its practical utility as a basis for implementing model-based flipping on RHex is limited.

Fortunately, it turns out that reintroducing Coulomb friction at the toes but maintaining the assumption of massless legs still yields a model wherein the typical range of operation for RHex's flipping is found to be away from singularities. The models we describe in the following two sections constitute the basis for our experimental flipping controllers. 


\subsection{Frictional Dynamics with Sliding Body, Sliding Toe Contacts}

In this section, we present a model with massless legs and Coulomb friction, wherein both the leg and body contacts are sliding on the ground. Defining the configuration and unknown force vectors as

$$
\begin{aligned}
& \mathbf{v}:=\left[F_{1}, F_{2}, F_{3}, \ddot{\alpha}, F_{c}^{z}, \ddot{y}_{t}\right]^{T} \\
& \mathbf{c}:=\left[\alpha, y_{b}\right]^{T},
\end{aligned}
$$

yields a system with two degrees of freedom - the body pitch and the horizontal position of the tail. For each leg, we can write the moment balance equations as

$$
\left(l \cos \gamma_{i}+l \bar{\mu}_{i} \sin \gamma_{i}\right) F_{i}=-\tau_{i},
$$

where $\bar{\mu}_{i}:=-\mu_{t} \operatorname{sign}\left(\dot{y}_{i}\right)$ is the effective Coulomb friction coefficient and $\gamma_{i}$ corresponds to the toe angle as shown in Fig. 3. In the operational range of the flipping controller, these equations are solvable. However, there are interesting "jamming" singularities in the remaining parts of the state space, which we investigate in Sec. 3.8.

Similarly, force and moment balances for the body link yield

$$
\begin{gathered}
\bar{\mu}_{1} F_{1}+\bar{\mu}_{2} F_{2}+\bar{\mu}_{3} F_{3}-\overline{\mu_{b}} F_{c}^{z}-m \ddot{y}_{b}=0 \\
F_{1}+F_{2}+F_{3}+F_{c}^{z}-m \ddot{z}_{b}=m g \\
\sum_{i=1}^{3}\left(d_{i} \cos \alpha-d_{i} \bar{\mu}_{i} \sin \alpha\right) F_{i}+\left(\left(h+\overline{\mu_{b}} d\right) \sin \alpha\right. \\
\left.+\left(\overline{\mu_{b}} h-d\right) \cos \alpha\right) F_{c}^{z}-I \ddot{\alpha}=\sum_{i=1}^{3} \tau_{i},
\end{gathered}
$$

where, once again, system kinematics yields the body accelerations $\ddot{y}_{b}$ and $\ddot{z}_{b}$ as functions of $\ddot{\alpha}$ and $\ddot{y}_{t}$. As before, the combination of (3) and (4) yields the matrices $\mathbf{A}(\mathbf{c}), \mathbf{b}(\mathbf{c}, \dot{\mathbf{c}})$ and $\mathbf{D}(\mathbf{c})$.

\subsection{Frictional Dynamics with Sliding Body, Fixed Rear Toe}

The last model we consider still has massless legs and Coulomb friction, and corresponds to cases where the rear toe is stationary under the influence of stiction. This model is primarily motivated by the observed behavior of RHex's flipping, where the rear toe stops sliding following the liftoff of the front and middle pairs of legs. We incorporate this model into our feedback controller to be activated when the measured (or estimated) system state indicates that the rear toe is indeed stationary. Defining

$$
\begin{aligned}
& \mathbf{v}:=\left[\begin{array}{llllll}
F_{1} & F_{2} & F_{3} & \ddot{\alpha} & F_{c}^{z} & F_{1}^{y}
\end{array}\right] \\
& \mathbf{c}:=\alpha,
\end{aligned}
$$


leaves us with a system with one degree of freedom. The moment balance for the rear leg includes the unknown horizontal ground reaction force, yielding

$$
l \cos \gamma_{1} F_{1}+l \sin \gamma_{1} F_{1}^{y}=-\tau_{1},
$$

while the moment balance equations for the middle and front legs remain the same as (3). Finally, the balance equations for the body link take the form

$$
\begin{gathered}
F_{1}^{y}+\bar{\mu}_{2} F_{2}+\bar{\mu}_{3} F_{3}-\overline{\mu_{b}} F_{c}^{z}-m \ddot{y}_{b}=0 \\
F_{1}+F_{2}+F_{3}+F_{c}^{z}-m \ddot{z}_{b}=m g \\
\sum_{i=1}^{3} d_{i} \cos \alpha F_{i}-\sum_{i=2}^{3} d_{i} \bar{\mu}_{i} \sin \alpha F_{i}-d_{1} \sin \alpha F_{1}^{y} \\
+\left(\left(h+\overline{\mu_{b}} d\right) \sin \alpha+\left(\overline{\mu_{b}} h-d\right) \cos \alpha\right) F_{c}^{z}-I \ddot{\alpha}=\sum_{i=1}^{3} \tau_{i}
\end{gathered}
$$

Similar to the previous two models, system kinematics yields the body accelerations $\ddot{y}_{b}$ and $\ddot{z}_{b}$ as functions of $\ddot{\alpha}$ we use (6) and (7) to compute the matrices $\mathbf{A}(\mathbf{c}), \mathbf{b}(\mathbf{c}, \dot{\mathbf{c}})$ and $\mathbf{D}(\mathbf{c})$.

\subsection{Existence of Solutions and Leg Jamming}

It is not immediately obvious that the equations presented in Sects. 3.6 and 3.7 can be solved. In fact, a major singularity arises in computing the ground reaction forces on sliding legs using the moment balance equation (3). To illustrate the inconsistency, suppose that leg $i$ is sliding forward with $\dot{y}_{i}>0$ and the leg is within the friction cone with $\cot \gamma_{i}<\mu_{t}$. When $\tau_{i}<0$, the massless legs in our model require a positive vertical component for the ground reaction force, $F_{i}>0$. However, solution of the leg moment balance equation yields $F_{i}=-\tau_{i} /\left(l \cos \gamma_{i}-l \mu_{t} \sin \gamma_{i}\right)<0$, resulting in an inconsistency. Consequently, when the leg is sliding forward and is inside the friction cone, there are no consistent solutions for the unknown forces and accelerations.

It turns out that this problem is a special case of the well known Painlevé's problem of a rigid rod sliding on a frictional surface $[6,10]$. For certain parameter and state combinations, it is impossible to find any consistent set of finite forces and accelerations and one needs to seek impulsive solutions for the unknown quantities. This problem and its variations stimulated a large body of work in frictional collisions $[2,16,17,19]$, which hypothesize that the rigid rod would "jam" in such cases and start pivoting around its toe.

Fortunately, empirical evidence accumulated over months of physical experiments with the robot reveals that in the absence of dramatic external disturbances, RHex operates in regions of its state space away from these singularities. Starting from a stationary position, the front four legs always slide backward which guarantees a solution for the associated ground reaction forces. Furthermore, even though the rear legs usually slide forward, RHex's 
kinematics ensure that the orientation of the rear two legs is always outside the friction cone, yielding a consistent solution for the associated reaction forces. Finally, the body link always slides forward and admits a consistent solution once the toe reaction forces are identified.

\section{Model Based Control of Flipping}

Our goal in designing a model-based controller for flipping is to maximize the acceleration of the body pitch, while maintaining contact of the body endpoint with the ground and respecting actuator torque constraints. Depending on the measured (or estimated) state of the rear toe, the appropriate model is chosen among those presented in Sects. 3.6 and 3.7 in formulating the maximization problem. The resulting feedback controller implicitly defines a switching law based on the physical state of the rear toe, with no explicit discrete internal states. On RHex, direct measurement of toe stiction is not possible and we instead use an empirically designed estimator [15].

For both planar models, when the system is far from singular regions described in Sec. 3.8, the unknown forces and accelerations can be computed by directly solving (1), yielding

$$
\mathbf{v}=\mathbf{A}^{-1}(\mathbf{c}) \mathbf{b}(\mathbf{c}, \dot{\mathbf{c}})+\mathbf{A}^{-1}(\mathbf{c}) \mathbf{D}(\mathbf{c}) \boldsymbol{\tau} .
$$

Both constrained systems are underactuated and direct inversion of these dynamics to obtain torque solutions is generally not possible. Furthermore, our task is not specified in terms of particular choices of ground reaction forces and accelerations. Rather, we are interested in the (in)stability properties of particular degrees of freedom in the system, particularly the body pitch, as well as various constraints arising from our assumptions in Sec. 3.1. As a consequence, our controller is based on a constrained optimization formulation informed by the underlying dynamics.

\subsection{Constraints on Control Inputs}

The first set of constraints we impose in solving (8) are motivated by physical limitations of RHex's actuators. Torque limitations for the simplest, resistive model of a geared DC motor arise from the interaction between the back EMF voltage, the maximum available supply voltage and the armature resistance. Our model based controller is designed to respect constraints based on this simple model for each motor, yielding decoupled torque limits for each leg.

We introduce a second constraint to explicitly enforce body-ground contact throughout the progression of the remaining degrees of freedom. Fortu-

nately, this requirement is easily captured through the constraint $F_{c}^{z}>0$, an inequality that is linear in the input torques. 
Definition 1. For a particular state $\mathbf{q} \in \mathcal{Q}$, we define the corresponding set of allowable torques, $\mathcal{T}(\mathbf{q})$ as the set of all torque input vectors $\boldsymbol{\tau} \in \mathbb{R}^{3}$ such that

$$
\begin{gathered}
F_{c}^{z}(\mathbf{q}, \boldsymbol{\tau}) \geq 0 \\
\forall i, F_{i}(\mathbf{q}, \boldsymbol{\tau}) \geq 0 \\
\forall i, \tau_{i}^{\min }(\mathbf{q}) \leq \tau_{i} \leq \tau_{i}^{\max }(\mathbf{q})
\end{gathered}
$$

\subsection{Maximal Thrust Control}

For both models of Sec. 3.6 and 3.7, the solutions for $\ddot{\alpha}$ and $\ddot{y_{t}}$ are continuous functions of the input torques. For any given state, this functional relationship is defined through our hybrid toe contact model and the solutions for the ground reaction forces. As a consequence, the problem of choosing hip controls to maximize thrust becomes a constrained optimization problem over the allowable input torque space.

Definition 2. Given the current state $\mathbf{q} \in \mathcal{Q}$, we define the maximal torque input $\boldsymbol{\tau}^{*}$ as the torque vector that yields the maximum pitch thrust:

$$
\boldsymbol{\tau}^{*}(\mathbf{q}):=\underset{\boldsymbol{\tau} \in \mathcal{T}(\mathbf{q})}{\operatorname{argmax}}(\ddot{\alpha}(\mathbf{q}, \boldsymbol{\tau})) .
$$

Fortunately, the solutions of (8) depend linearly on the input torques. Consequently, the constraints in Defn. 1 as well as the objective function, $\ddot{\alpha}(\mathbf{q}, \boldsymbol{\tau})$, are linear in the input torques as well. As a result, standard linear programming techniques can be employed to identify efficiently the maximal torque solution $\tau^{*}$. In particular, we use a simple geometric solution that exploits the low dimension and the largely decoupled structure of the constraints [11].
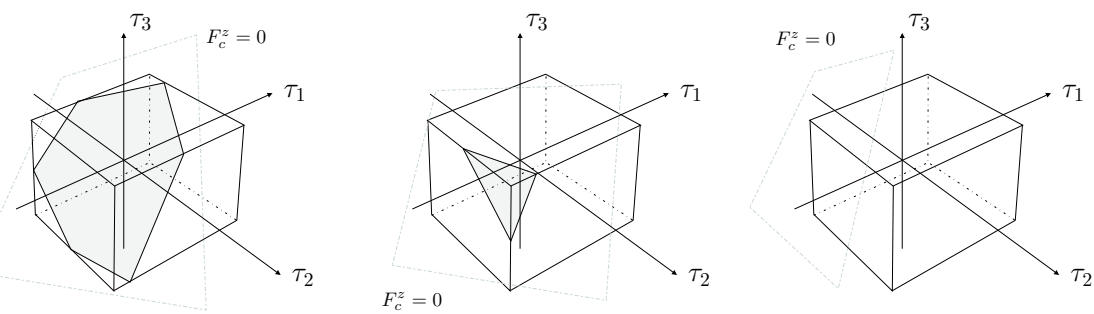

Fig. 5. All possible arrangements of volumes for motor torque limits and the body liftoff constraint. Simple geometric analysis yields the solution that yields the maximum thrust, while satisfying all the constraints.

As illustrated in Fig. 5, motor torque limits can be expressed as an axisaligned constraint volume in the hip torque space, which can then be intersected with the half-space resulting from the inequality constraint of (9). The 
optimal solution can be obtained by simply evaluating the objective function on the vertices of the resulting volume. There are only three possible arrangements of these volumes corresponding to different geometric arrangements of the cube relative to the body constraint half space. The only case where there are no solutions that satisfy all the constraints is when these volumes do not intersect at all, in which case we prioritize the motor torque limit constraint and choose a solution that yields the smallest vertical force on the tail.

\section{Experimental Results}

\subsection{Experimental Platform}

The most recent version of the robot, RHex 1.5, adopted for the present experiments, has a rigid body that measures $50 \times 20 \times 15 \mathrm{~cm}$, and houses all the computational and motor control hardware. The total weight of the robot is roughly $8.5 \mathrm{~kg}$. Each of RHex's six leg is directly actuated by a Maxon RE118751 $20 \mathrm{~W}$ brushed DC motor combined with a Maxon 114473 two-stage $33: 1$ planetary gear, delivering an intermittent stall torque of $6 \mathrm{Nm}$ at $24 \mathrm{~V}$. We achieve approximate software torque control at $1 \mathrm{KHZ}$ by adopting an approximate feedforward model of the motor/amplifier stacks ${ }^{3}$ - a variant of the model described in [8]. RHex 1.5 also incorporates a three-axis gyro for inertial sensing of the body orientation in addition to the motor encoders.

The legs on the current RHex are monolithic pieces of compliant fiberglass, attached to motor shafts through aluminum hip fixtures. Each of the legs in the set used for the experiments in this paper is roughly $16.5 \mathrm{~cm}$ long, weighs $80 \mathrm{~g}$ and has a radial compliance ${ }^{4}$ of $1900 \mathrm{~N} / \mathrm{m}$ [9].

\subsection{Thrust Phase Model Performance}

In this section, we present experimental data to establish the performance of our thrust controller on linoleum, a slippery surface with relatively consistent frictional properties. For the experiments presented in this section, we fixed the friction coefficient for the body contact as $\mu_{b}=0.4$, based on ranges indicated in [5] for plastic on linoleum type surfaces. The maximal thrust controller of Sec. 4.2 explicitly minimizes the ground reaction force on the tail. Hence, the frictional force on the tail is also very small, significantly decreasing the importance of our particular choice for the associated coefficient of friction on the overall performance of the controller.

In contrast, in order to estimate the much more important toe friction coefficient and assess the corresponding model performance, we ran a number

\footnotetext{
${ }^{3}$ See [15] for details.

${ }^{4}$ Even though compliance is critical in RHex's dynamic locomotion performance, it is not nearly as dominant for the flipping behavior.
} 
of experiments using approximate measurements of RHex's kinematic and dynamic parameters for different settings of the toe friction coefficient $\mu_{t}$. Four runs were recorded for 15 different settings in the range $\mu_{t} \in[0.1,0.6]$. Figure 6 displays a sequence of snapshots for one of these experiments, extracted from the high speed video footage.

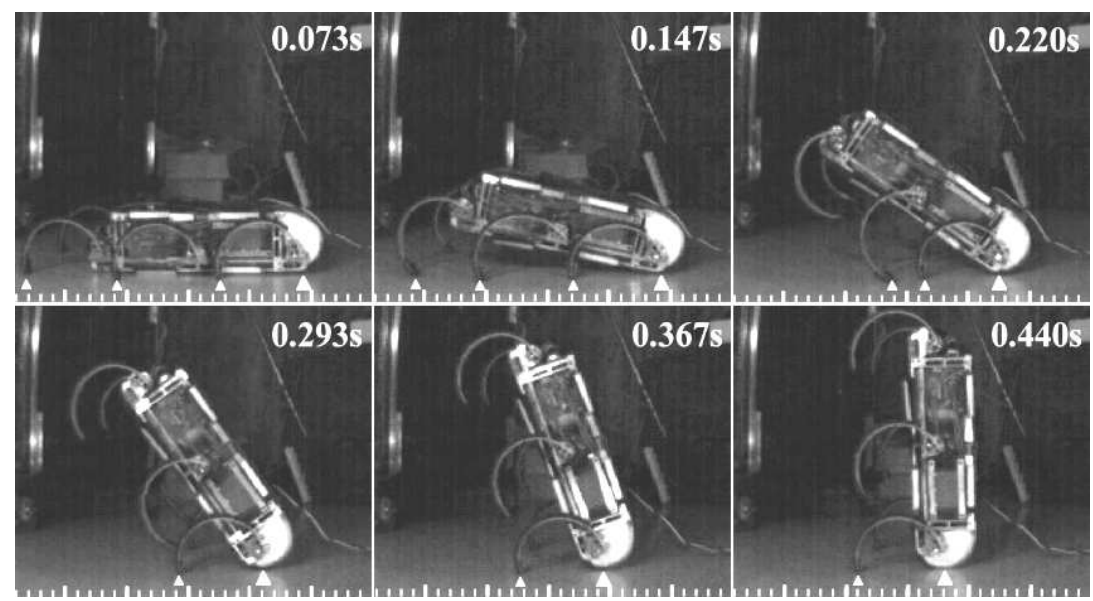

Fig. 6. Sequence of snapshots during flipping on linoleum with $\mu_{t}=0.39$, for the period where the maximal thrust controller is active. Subsequent frames in which the robot falls back and recovers are not included. White arrows indicate contact points for the toes and the body

The best model performance was obtained for $\mu_{t}=0.39$ (see Fig. 7) with a mean RMS error of $0.4 \mathrm{rad} / \mathrm{s}$ between model predicted pitch velocity and experimental measurements; surprisingly small considering various levels of approximations used in our model, including the inaccuracies in the kinematic and dynamic parameters, unmodeled leg compliance and the approximate torque control.

These results suggest that our model provides an accurate representation of the thrust phase. Nevertheless, there are a number of inaccuracies in its prediction, visible in the pitch acceleration plots. Most significantly, our model fails to predict the large overestimation of the initial acceleration and the subsequent, relatively large oscillations in the measured acceleration.

We believe that the origin of both discrepancies is the compliance in RHex's legs. The initially uncompressed legs introduce some delay in responding to the torque commands, resulting in a delayed acceleration of the pitch. A similar effect is visible subsequent to the liftoff of the middle legs which also causes oscillations due to the sudden loading of the rear legs.

Nevertheless, most of these differences do not significantly influence the average performance of the model. In addition to the accurate prediction of 

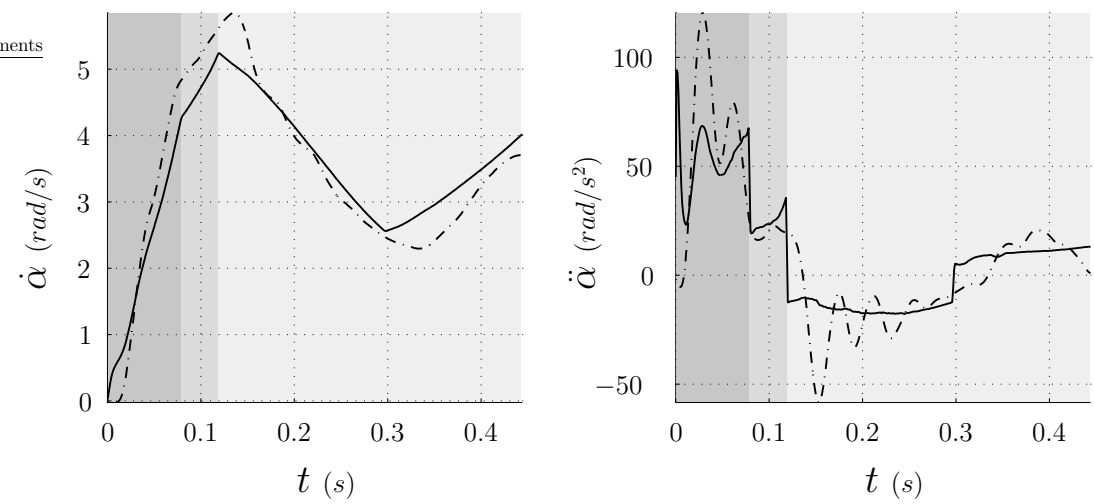

Fig. 7. Model predictions for the pitch velocity(left) and acceleration(right) compared to the experiment with best model performance $\left(\mu_{t}=0.39\right)$. Solid lines indicate model prediction whereas dashed lines show the actual measurements. Shaded regions indicate different number of legs in contact with the ground: dark(3), middle(2) and light(1)

the pitch velocity, the robot successfully keeps its tail on the ground and consistently performs flips on linoleum in a single thrust (see Fig. 6).

\section{Conclusion}

In robotic locomotion research, autonomy is likely to impose some of the most demanding constraints on design and limitations on behavior. It is very difficult, often impossible to achieve in systems otherwise designed for nonautonomous operation. RHex, our hexapedal platform, demonstrated that autonomy as a design goal can achieve significant advances in real world performance and robustness.

In this paper, we present a new controller to implement self-righting behavior on RHex, which is perhaps the simplest instance of maneuverability other than locomotion itself. To this end, we investigate different options for modeling the flipping behavior, each with different practical as well as analytic properties. In doing so, we review a number of models we had introduced in earlier work, and highlight their analytical properties and singularities with respect to their potential implementation on RHex. A new, simpler model still offers the advantages of some of these earlier approaches in its singularity properties while being much more feasible for practical deployment. The main focus of the paper is to identify common fundamental problems that arise in models of dynamical systems with multiple contacts and external actuation, with an emphasis on the implications of these problems in the design of feedback controllers. 
Our modeling efforts reveal two distinct types of singularities in the models proposed for the self-righting behavior. The simplest approach with nonfrictional contacts and massless legs results in singular configurations which are fairly straightforward to characterize and yield physically inaccurate model predictions. The same problem persists even when we recover physical accuracy by introducing leg masses at the expense of model simplicity due to nonlinear kinematics and algorithmic complexities in determining contact state.

In contrast, a new class of singularities appear when we consider contacts with Coulomb friction, resulting in ranges of inconsistent states wherein one cannot find solutions for the dynamics. It turns out that this class of singularities is an instance of the relatively well-known Painlevé's problem of a rigid rod sliding on a frictional surface. Our flipping controller overcomes the problematic consequences of this singularity by ensuring that the progression of the robot state throughout the behavior stays away from singular regions. The feasibility of this approach is demonstrated by systematic experiments on RHex, which both demonstrate the performance of the controller and show that model predictions closely match with experimental measurements during flipping.

In parallel with most of the literature in modeling multiple contacts, our approach is to present domain specific characterizations of various models and equally specialized solutions for their usage in designing feedback controllers. However, extensions to the flipping behavior such as uninterrupted rolling or handstands are likely to require a much better analytical understanding of the various proposed models. In particular, a more unified characterization of singularities for generic classes of models with multiple contacts is critical for the ability to design robust controllers that explicitly avoid or exploit singular configurations. We believe that understanding and resolving fundamental dynamical properties of robotic systems arising from their morphology in conjunction with multiple intermittent contacts will be instrumental in the design and deployment of robust and practical controllers for such systems.

\section{Acknowledgments}

We thank Prof. Matt Mason for his insight on friction models and the connection to Painlevé's problem. This work was supported in part by DARPA/ONR Grant N00014-98-1-0747.

\section{References}

1. R. Altendorfer, N. Moore, H. Komsuoglu, M. Buehler, H. B. Brown Jr., D. McMordie, U. Saranli, R. J. Full, and D. E. Koditschek. RHex: A Biologically Inspired Hexapod Runner. Autonomous Robots, 11:207-213, 2001.

2. D. Baraff. Coping with Friction for Non-penetrating Rigid Body Simulation. Computer Graphics, 25(4):31-40, 1991. 
3. P. Fiorini and J. Burdick. The Development of Hopping Capabilities for Small Robots. Autonomous Robots, 14(2-3):239-254, May 2003.

4. E. Hale, N. Schara, J. W. Burdick, and P. Fiorini. A Minimally Actuated Hopping Rover for Exploration of Celestial Bodies. In Proceedings of the IEEE International Conference On Robotics and Automation, pages 420-7, San Francisco, CA, April 2000.

5. L. S. Marks. Marks' standard handbook for mechanical engineers. McGraw-Hill, New York, 1996.

6. M. T. Mason. Mechanics of Robotic Manipulation. MIT Press, 2001.

7. L. Matthies, Y. Xiong, R. Hogg, D. Zhu, Rankin A., B. Kennedy, M. Hebert, R. Maclachlan, C. Won, T. Frost, G. Sukhatme, M. McHenry, and S. Goldberg. A Portable, Autonomous, Urban Reconnaissance Robot. In Proceedings of The 6th International Conference on Intelligent Autonomous Systems, Venice, Italy, July 2000.

8. D. McMordie, C. Prahacs, and M. Buehler. Towards a Dynamic Actuator Model for a Hexapod Robot. In Proceedings of the IEEE International Conference On Robotics and Automation, 2003. accepted for publication.

9. E. Z. Moore, D. Campbell, F. Grimminger, and M. Buehler. Reliable Stair Climbing in the Simple Hexapod RHex. In Proceedings of the IEEE International Conference On Robotics and Automation, volume 3, pages 2222-2227, Washington, DC., May 2002.

10. P. Painlevé. Sur le lois de frottement de glissement. C. R. Acad. Sci. Paris, 121:112-115, 1895.

11. U. Saranli. Dynamic Locomotion with a Hexapod Robot. PhD thesis, Computer Science and Engineering. The University of Michigan, 2002.

12. U. Saranli, M. Buehler, and D. E. Koditschek. RHex: A Simple and Highly Mobile Robot. International Journal of Robotics Research, 20(7):616-631, July 2001.

13. U. Saranli and D. E. Koditschek. Design and Analysis of a Flipping Controller for RHex. Technical Report CSE-TR-452-02, UM, Ann Arbor, MI, 2001.

14. U. Saranli and D. E. Koditschek. Back Flips with a Hexapedal Robot. In Proceedings of the IEEE International Conference On Robotics and Automation, volume 3, pages 128-134, Washington, DC., May 2002.

15. U. Saranli, A. Rizzi, and D. E. Koditschek. Model-Based Dynamic Self-Righting Maneuvers for a Hexapedal Robot. International Journal of Robotics Research, 2004. Accepted for publication.

16. D. E. Stewart. Convergence of a Time-Stepping Scheme for Rigid-Body Dynamics and Resolution of Painleve's Problem. Archive for Rational Mechanics and Analysis, 145(3):215-260, 1998.

17. D. E. Stewart and J. C Trinkle. Dynamics, Friction, and Complementarity Problems. In M. C. Ferris and J. S. Pang, editors, Complementarity and Variational Problems, pages 425-439. SIAM, 1997.

18. E. Tunstel. Evolution of Autonomous Self-Righting Behaviors for Articulated Nanorovers. In Proceedings of the 5th International Symposium on Artificial Intelligence, Robotics and Automation in Space, pages 341-6, Noordwijk, The Netherlands, June 1999.

19. Y. Wang and M. Mason. Two Dimensional Rigid Body Collisions with Friction. Trans ASME; J Applied Mechanics, 59(3):635-642, September 1992. 